\title{
Mechanical property and biocompatibility of co-precipitated nano hydroxyapatite-gelatine composites
}

\author{
Huirong LE ${ }^{a,{ }^{*}}$, Kiruthika NATESAN $^{b}$, Sundaram PRANTI-HARAN ${ }^{c}$ \\ ${ }^{a}$ School of Marine Science and Engineering, Plymouth University, United Kingdom \\ ${ }^{b}$ Peninsular Schools of Medicine and Dentistry, Plymouth University, United Kingdom \\ ${ }^{c}$ School of Engineering, Physics and Mathematics, University of Dundee, United Kingdom
}

Received: April 01, 2015; Revised: April 20, 2015; Accepted: May 03, 2015

(C) The Author(s) 2015. This article is published with open access at Springerlink.com

\begin{abstract}
Uniform and well dispersed nano hydroxyapatite (HA)-gelatine composites were obtained by co-precipitation of hydroxyapatite and denatured calf skin collagen. The process allowed much higher concentration of hydroxyapatite to be produced over conventional hydrothermal process to improve the productivity. The effect of gelatine on the morphology, mechanical properties, and biocompatibility of hydroxyapatite particles was investigated. Fibroblast cell tests of the consolidated hydroxyapatite-gelatine composites showed that the composites have excellent biocompatibility.
\end{abstract}

Keywords: biomaterials; nanocomposites; hydroxyapatite (HA); gelatine; biocompatibility

\section{Introduction}

An extremely specialized supporting framework of the body protecting the vital organs is bone. Natural bone is a composite material composed of hybrid organic and inorganic constituents. It has a varied arrangement of material structures at many length scales which work in concert to perform diverse mechanical, biological, and structural properties. The uninterrupted growth in the life expectancy of population has led to continuous conditions which are prone to injury causing severe damage to the individual. While bone is inherently capable of regeneration, complications such as excessive bone loss impede healing, necessitating the use of bone grafts [1]. Bone is one of the most frequently implanted tissues in human body, second only behind blood. Due to the advances in biomaterial research, it is now possible to artificially fabricate

* Corresponding author.

E-mail: huirong.le@plymouth.ac.uk materials that mimic the structure of natural bone to be used as bone replacement.

Hydroxyapatite (HA) is the primary inorganic substance in natural bone and has been studied extensively for medical applications due to its excellent bioactivity and biocompatibility. However, pure sintered HA has low fracture toughness and low degradation properties which limit its use to only non-stress applications [2,3]. Previous research [4-11] has shown that a composite can be formed that closely resembles the structure of natural bone tissue by combining a mixture of natural macromolecules such as collagen and synthetic hydroxyapatite particles by hydrothermal process. Although the resulting composites have been proven to have good biocompatibility, they suffer from low modulus.

In order to enrich the characteristics of individual components, organic-inorganic composites can be synthesised through a hybridization route to build a harmonious balance between strength and toughness. One technique of achieving this is the sol-gel process 
which allows the reinforcement of organic polymers within inorganic calcium phosphates producing organic-inorganic hybrids [12]. Fabrication route of HA has a strong influence on the crystal morphology. Even with the sol-gel process, different shapes and sizes ranging from nano to micro particles of HA can be produced by altering the reaction rate, $\mathrm{pH}$, and temperature along with the presence or absence of surfactants. To be able to control nucleation and crystal morphology, many researchers have attempted to use various surfactants and polymers [13-15] and have successfully grown HA crystals with varying morphology.

Natural macromolecules such as gelatine and chitosan are commonly used as binders in biomedical applications. These polymers have been used as binders for HA particles, or HA have been precipitated in their presence using various techniques [16-18]. Gelatine being a denatured form of collagen has many attractive properties such as biocompatibility, plasticity, and adhesiveness $[16,19]$. However, there is still a lack of understanding on the effects of these molecules on HA crystal morphology, its mechanical strength, and biocompatibility.

The hard tissue of bone is made up of biological apatite which is a non-stoichiometric hydroxyapatite. Natural bone hydroxyapatite has trace amount of several other ions. The precipitation route of HA has a prominent effect on the morphology, shape, and size of HA. Even small differences in stoichiometry, crystallinity, or morphology are expected to lead to different chemical, biological, and physical behaviours of the material [20-22]. Previous study by the authors investigated the effect of $\mathrm{pH}$ and temperature on the morphology of sol-gel co-precipitated HA [23]. X-ray diffraction (XRD) analysis confirmed that the material obtained is mainly $\mathrm{HA}$ with trace of brushite $\left(\mathrm{CaHPO}_{4} \cdot 2 \mathrm{H}_{2} \mathrm{O}\right)$. Under desirable conditions, a plate-like structure was developed by self-assembly of columnar crystals. This paper is intended to further report on the effect of the addition of gelatine on the morphology and dispersion of HA particles. The biocompatibility of the obtained HA-gelatine composites from this process will also be reported.

\section{Experimental methods}

\section{1 Hydroxyapatite and hydroxyapatite-gelatine composite preparation}

A diagrammatic representation of the precipitation process developed previously by the authors is shown in Fig. 1. In this method, $0.2 \mathrm{~mol} / \mathrm{L}$ calcium chloride and $0.12 \mathrm{~mol} / \mathrm{L}$ ammonium hydrogen phosphate solutions were dropped simultaneously into a reaction vessel at a controlled rate of about $0.5 \mathrm{~L} / \mathrm{h}$ using valve controlled burettes. The solution was stirred constantly using magnetic stirrer to maintain constant temperature and facilitate the reaction. The product first appeared as a gel and then milky suspension with more solutions being added. The $\mathrm{Ca}$ to $\mathrm{P}$ ratio was maintained at 1.67 by using equal volumes of $0.2 \mathrm{~mol} / \mathrm{L}$ calcium containing solution and $0.12 \mathrm{~mol} / \mathrm{L}$ phosphate containing solution. The effect of temperature and $\mathrm{pH}$ on the morphology of hydroxyapatite was reported by the authors in previous work [23].

To fabricate hydroxyapatite-gelatine composites, calf skin collagen (Sigma-Aldrich, Collagen I) was first dissolved in $0.5 \mathrm{~mol} / \mathrm{L}$ acetic acid at $40{ }^{\circ} \mathrm{C}$ under continuous stirring. After collagen becoming a uniform gel, the solution was titrated with $0.5 \mathrm{~mol} / \mathrm{L}$ sodium hydroxide solution $(\mathrm{NaOH})$ to the desired $\mathrm{pH}$. Hydroxyapatite was precipitated in the presence of the dissolved collagen with a target collagen to HA ratio of $1: 10$.

The $\mathrm{pH}$ values of all the reactions were maintained at 9-9.5 using $0.5 \mathrm{~mol} / \mathrm{L}$ sodium hydroxide $(\mathrm{NaOH})$ or $0.5 \mathrm{~mol} / \mathrm{L}$ acetic acid $\left(\mathrm{CH}_{3} \mathrm{COOH}\right)$. The former was used when there was a drop in $\mathrm{pH}$ during the reaction and the latter was used when the $\mathrm{pH}$ was observed to increase. The magnetic stirrer was kept at constant speed of $300-500 \mathrm{rpm}$ for all the reactions. The reaction vessel temperature was kept at nominal $40{ }^{\circ} \mathrm{C}$.

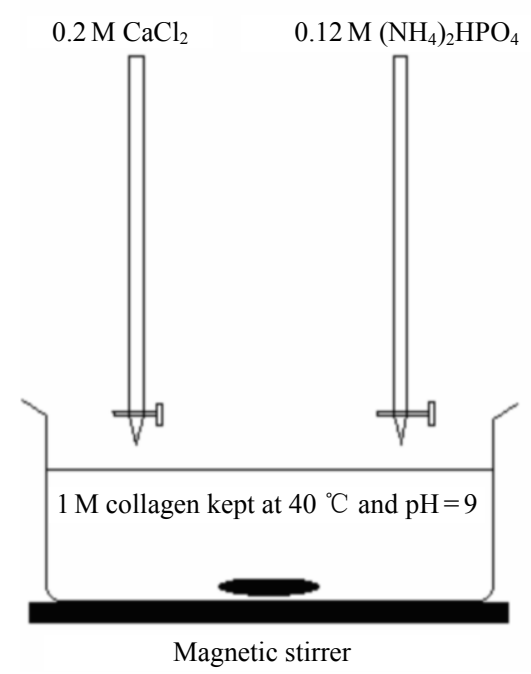

Fig. 1 Co-precipitation of hydroxyapatite-gelatine composites. 
Once the reaction was completed, the reaction vessel containing the product was moved into water bath to age at $40{ }^{\circ} \mathrm{C}$ for $24 \mathrm{~h}$.

After aging, a small volume of the aged precipitates was removed and taken for analysis. The suspension was then filtered to remove excessive water and free ions using vacuum assisted filtering system. The composite suspension was poured into the collection beaker with a filter paper. Once the excessive water was removed from the filter, the next step was to rinse the precipitates using deionised water for several times. Atmospheric impurities and other unwanted ions were removed from the composites with fresh deionised water, including chloride $\left(\mathrm{Cl}^{-}\right)$, sodium ions $\left(\mathrm{Na}^{+}\right)$, and ammonium ions $\left(\mathrm{NH}_{4}^{+}\right)$, etc.

Whilst applying efficient means of filtering the precipitates, only majority of the free water content was removed. As there was water trapped inside the precipitates, vacuum filtration was not enough to remove all of the water content. The precipitate paste was placed into a Petri dish and then kept inside an incubator. The incubator was set to a constant temperature of $40-60{ }^{\circ} \mathrm{C}$. The weight of the precipitates was taken at regular intervals, so the drying process was continued until a constant weight was achieved.

\section{2 Sample preparation for mechanical tests}

In order to examine the mechanical modulus and strength of the composites, the dried composite precipitates were mixed with deionized water so that the liquid to solid ratio was about 1:1. After regular monitoring on the weight of each of the precipitates that were dried in the oven, the precipitates were taken out at water to solid ratio of about 1:2. A number of test blocks with dimension of $24 \mathrm{~mm} \times 4 \mathrm{~mm} \times 2 \mathrm{~mm}$ were prepared from each precipitate using a Tufnol mould with a rectangular slot. For each composite, the weight for one sample piece was calculated and then placed inside the mould. The load was applied using a hydraulic press. The load was gradually increased to $5 \mathrm{kN}$ (equivalent to a pressure of $50 \mathrm{MPa}$ ) and held for $1 \mathrm{~min}$. This would compress the sample, thereby squeezing out the excessive water content that would not have been removed by incubation. After $1 \mathrm{~min}$ dwell, the load was gradually increased to $10 \mathrm{kN}$ (equivalent to a pressure of $100 \mathrm{MPa}$ ) and then held for another $1 \mathrm{~min}$. By this point the composites would have been suitably compressed to produce a strong rectangular solid block. The solid sample was then popped out from the mould.

\section{3 Crystallographic and microstructural characterizations}

The phase constituents of each precipitate were examined using XRD. Certain amount of dried precipitate block was ground into powder and then pressed into flat sample. X-ray powder diffraction patterns were measured on Rigaku D/max2500 X-ray diffractometer with $\mathrm{Cu} \mathrm{K} \alpha$ radiation at a scanning speed of $6\left(^{\circ}\right) / \mathrm{min}$ in the range of $10^{\circ}-80^{\circ}$. The operating voltage and current of this XRD machine were $40 \mathrm{kV}$ and $200 \mathrm{~mA}$, respectively. The phases of each precipitate were determined by comparing the XRD patterns with JCPDF database.

Scanning electron microscopy (SEM) was used for studying the morphology of the precipitates fabricated. The machine used was Philips XL 30 ESEM (Dundee). The samples were mounted on aluminium stubs. The surface of the stub had carbon adhesives which held the samples in place. Then these stubs were moved into a sputter coater. The dried blocks were coated with gold/palladium up to $30 \mathrm{~nm}$ of thickness in order to dissipate the fast impinging electrons on the specimens during SEM operation.

\section{4 Fibroblast cell tests}

For the biocompatibility tests of the materials to be carried out, the obtained hydroxyapatite-gelatine composite solid blocks were broken into small test beads of about $4 \mathrm{~mm} \times 4 \mathrm{~mm}$.

The test beads were sterilised by soaking them in ethanol for $4 \mathrm{~h}$. The samples were then removed using sterile tweezers and placed in sterile beakers. The beakers were heated to $80{ }^{\circ} \mathrm{C}$ to dry the ethanol soaked samples. Sterile Petri dishes were coated with a layer of silicone to encourage cell growth on the samples rather than the surface of the Petri plate. The cell growth media consisted of fetal bovine serum (FBS) and antibiotic-antimycotic (ABAM) solution. FBS medium provided good nutrient to the cells while the ABAM was used to prevent bacterial and fungal infections.

Once the samples were dry, they were moved into the cell growth medium. In this process two volumes of bovine serum of the same concentration were used. The composites were kept in one volume of bovine serum, and in the other volume of the bovine serum 
fibroblasts of rat tendon were cultured. About $15 \mu \mathrm{L}$ of the cultured rat tendon fibroblasts was taken and applied onto the composite sample in the Petri dish. The test was performed for 7 days, with nutrient being replenished every $24 \mathrm{~h}$. The samples were critical point freeze dried with liquid nitrogen and placed in a freeze drier overnight. They were then mounted on stubs and painted in silver paint (Agar Scientific Ltd.). These were placed in an incubator at $40{ }^{\circ} \mathrm{C}$ for $20 \mathrm{~min}$ followed by gold coating. These samples were subsequently considered stable and were viewed under SEM.

\section{Results and discussions}

\section{1 Microstructure of hydroxyapatite}

The SEM image of hydroxyapatite crystals obtained at $40{ }^{\circ} \mathrm{C}$ and $\mathrm{pH}=9$ by controlled precipitation process is shown in Fig. 2. The hydroxyapatite crystals are typical of interlocked plates and scattered nano-particles. The width of the plates is about $2 \mu \mathrm{m}$ and the thickness is about $80 \mathrm{~nm}$ according to SEM measurements. It can be seen that HA has developed distinctive lenticular crystals and subsequent planar assembly during the aging process.

In order to explain this, various factors such as preparation time and aging period should be taken into consideration. This significant difference in the morphology of the crystals could arise as a result of the mixing rate of the precursor solutions as it has a direct impact on the nucleation of HA. Slow mixing of calcium and phosphate precursors in this process results in less nucleation sites and the growth of primary crystals in lenticular form [24].

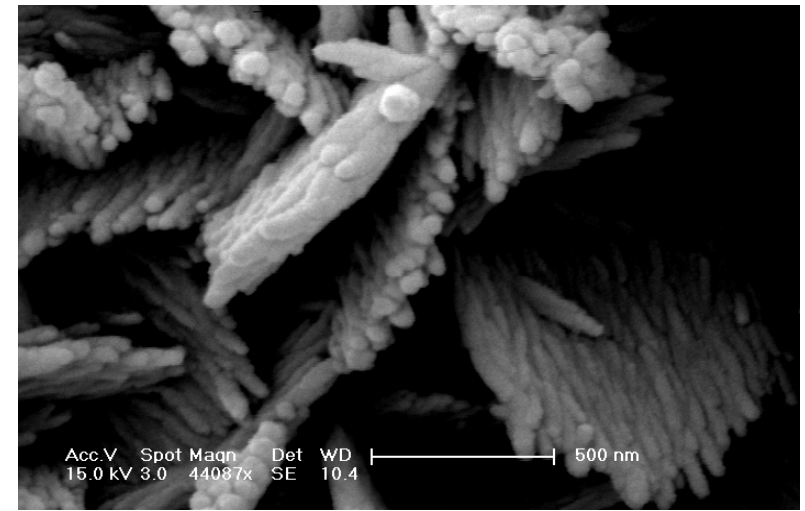

Fig. 2 Closer view of HA crystals prepared at $40{ }^{\circ} \mathrm{C}$ and $\mathrm{pH}=9$ by slow precipitation.

\section{2 Effect of gelatine on the morphology of HA}

The morphology of the HA-gelatine composites prepared at $40{ }^{\circ} \mathrm{C}$ and $\mathrm{pH}=9$ is shown in Fig. 3. It is noted that collagen fibres are not visible even under high resolution SEM. It is believed that the collagen fibres have been denatured into gelatine in acetic acid solution under the temperature applied $[25,26]$. The morphology of hydroxyapatite particles is different from that of pure hydroxyapatite prepared under the same conditions. The particles in Fig. 3 are typical of spherical granules of about 10-100 nm compared to the particles of $80-1000 \mathrm{~nm}$ shown in Fig. 2. This is inferring that the nucleation rate of HA crystals is much higher due to the presence of gelatine molecules in the suspension. This is a result of lower activation energy required in heterogeneous nucleation of hydroxyapatite crystals on the polypeptide structure of gelatine as described in Ref. [27]. The primary spherical clusters fuse together to form a much more compact and interconnected material with the organic component acting as an adhesive. There is lower probability to form large agglomerates or plate-like structure because the surface energy of the particles is smaller. Thus a uniform and well dispersed nanostructure is obtained.

XRD analysis shown in Fig. 4 shows that hydroxyapatite precipitated in the gel has more significant peak widening than that prepared in water. This confirms that the grain size is smaller in the presence of gelatine molecules due to heterogeneous nucleation as discussed above.

\section{3 Mechanical behaviour of hydroxyapatite-gelatine composites}

The pressed bar samples were tested using three point

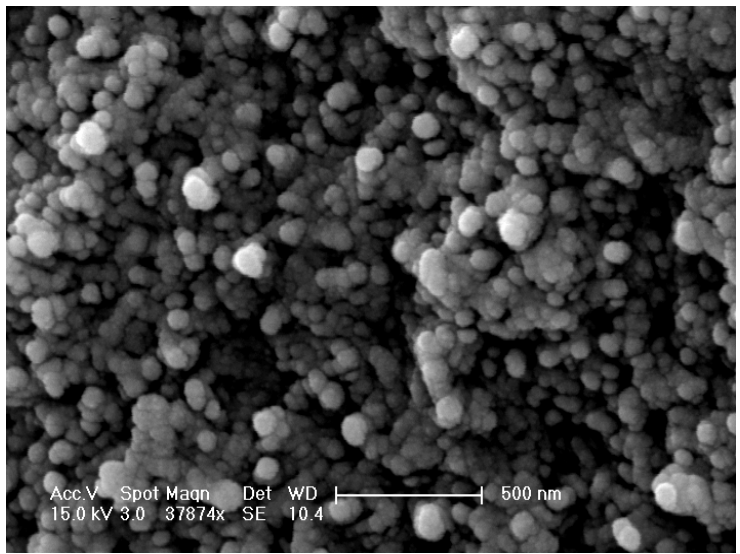

Fig. 3 Morphology of HA-gelatine composites. 


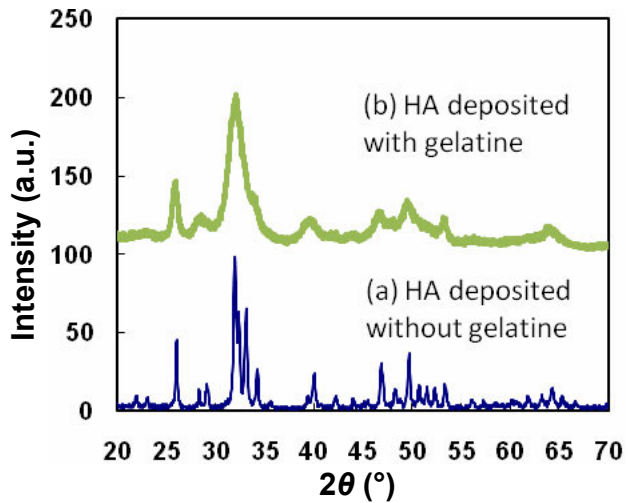

Fig. 4 XRD graphs of (a) HA deposited without gelatine and (b) HA deposited with gelatine.

bending tests with a $24 \mathrm{~mm}$ span. The graph in Fig. 5 is the force-displacement curve obtained for a test block of the prepared composites. From the graph it could be seen clearly of the gradual and uniform increase of the applied force in loading. The dimensions of all the test blocks were measured before the process, in order to determine the fracture strength of the material. The deflection starts from $0.02 \mathrm{~mm}$ and runs through a distance up to $0.14 \mathrm{~mm}$, where the peak load occurs. There are no signs of artefacts or disturbances observed in the graph. The fracture of the test block occurs at a maximum load of 7.9 N. Six samples were tested. The average fracture strength of hydroxyapatite-collagen composited is about 15.7 MPa. This is at the lower end of the strength of cortical bones, but higher than cancellous bones [26]. It is believed that the collagen molecules might have been denatured and become gelatine during the fabrication process which is responsible for rather modest fracture strength. Nevertheless, the composites exhibit some extensibility after a crack is initiated. This is owing to the stretching of the organic constituencies in the composites.

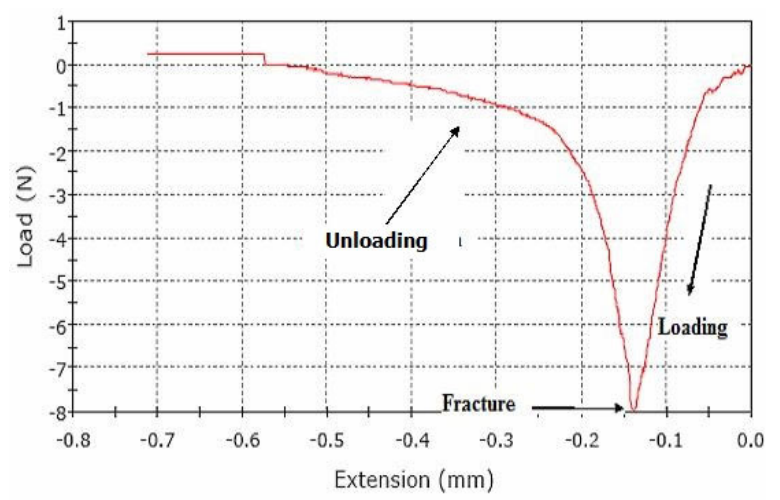

Fig. 5 Force-displacement curve of three point bending test of $10 \%$ collagen composites.

\section{4 Cytocompatibility}

The bovine growth medium provides good source of nutrient for the rat tendon fibroblasts to grow. The SEM observations (Figs. 6-9) show that many fibroblast cells are clearly visible on all the specimens after 7 days of tests. The cells are large and flat with numerous pseudopods. They are highly interconnected and healthy growth is observed on all the samples. These images are clear evidence of cell attachment and proliferation on the surface of the material. It is also noted that the cells could grow on irregular fractal surface of the samples such as ridges and deep cavities (Figs. 6-8). It is evident that the material is biocompatible and promotes cell attachment and growth. The denaturisation of collagen does not have any negative impact on the cell adhesion to the surface of the composites. Once these fibroblasts start to grow, they tend to stick to the surface of the composite samples. If there is any contamination of the composite samples which would produce toxic effects on the living tissues, the entire bovine serum growth media

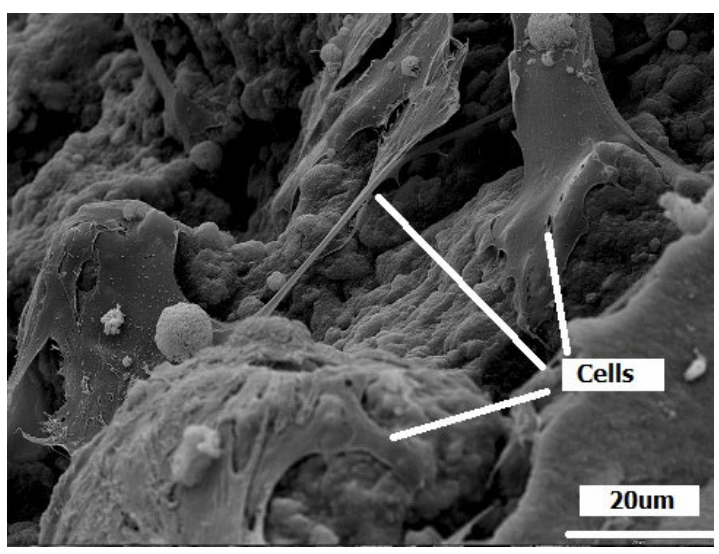

Fig. 6 Cell attachment to curved composite surface of HA-gelatine composites.

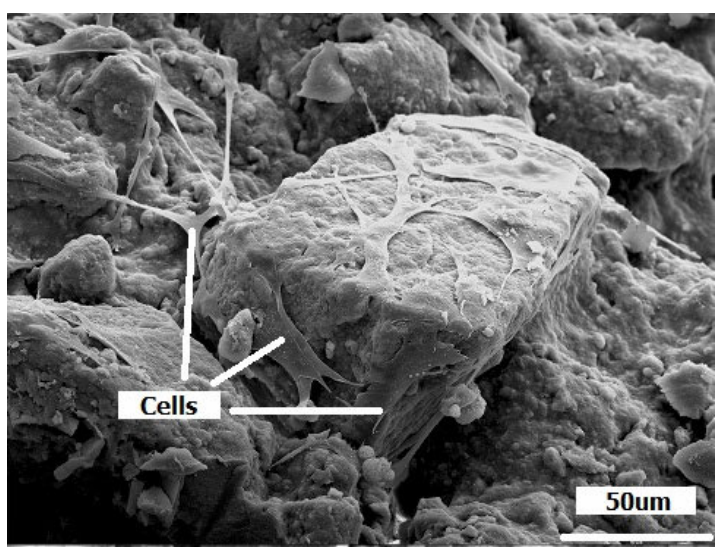

Fig. 7 Cell growth on surface ridges of HA-gelatine composites. 


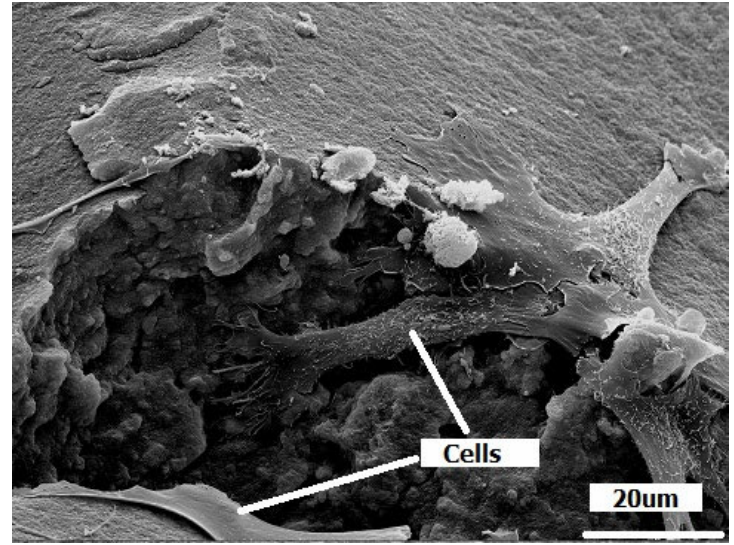

Fig. 8 Cell growth into surface cavities of HA-gelatine composites.

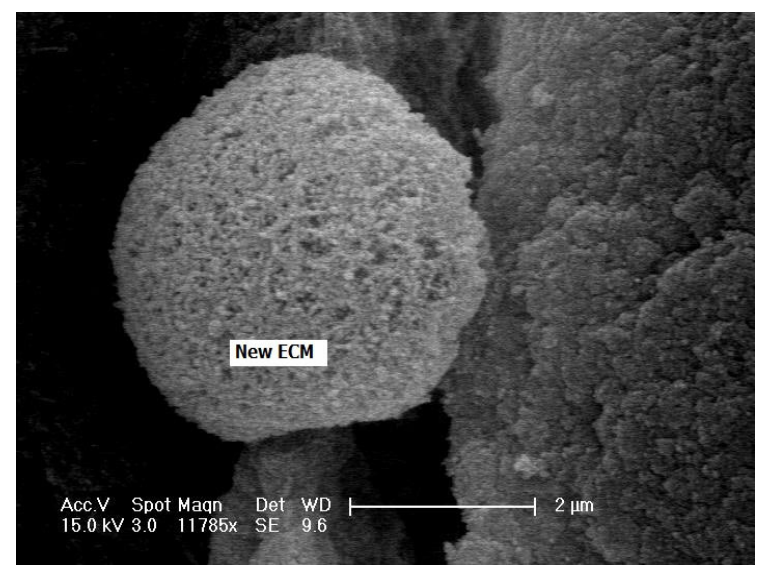

Fig. 9 Material regeneration during fibroblast cell tests.

becomes toxic. This would lead to the death of the cells that are introduced into the growth media.

The growth of new extracellular matrix (ECM) during the cell tests is also noted in the SEM image shown in Fig. 8. One can see the lighter globules attach to the cells and the surface of the test material. The morphology of these globules is very different from the original material. At nearly 12000 times of magnification (Fig. 9), it becomes clear that the globules are porous clusters of nano-particles and have a diameter of about $3 \mu \mathrm{m}$. The morphology of the globules implies that it is a new precipitate when the material is submerged in the cell culture. One possible explanation is that the hydroxyapatite has dissolved gradually in cell culture and new precipitates of nano-crystals have occurred. These new precipitates could attach to the pseudopods developed on the cell surface to form a good attachment. This observation confirms that the HA-gelatine nanocomposites have the potential to regenerate new bone in desirable environment.

\section{Conclusions}

An efficient process based on high-concentration sol-gel precipitation was applied to the fabrication of hydroxyapatite and gelatine composites. The productivity was about two orders of magnitude higher than conventional hydrothermal process. In the presence of gelatine in the solution, uniform and well dispersed nano hydroxyapatite particles were obtained due to heterogeneous nucleation. Fibroblast cell tests on the composites showed excellent biocompatibility. Potential applications of the consolidated material include maxillofacial, skeletal bone grafts, and scaffolds for bone tissue engineering.

\section{Acknowledgements}

The authors would like to thank all the technicians in the School of Engineering, Physics and Mathematics, University of Dundee for their assistance in the manufacture of the mould and testing rig. The authors are also indebted to Dr. Kenneth Donnelly and Dr. Robert Keatch for constructive discussions and support.

Open Access: This article is distributed under the terms of the Creative Commons Attribution License which permits any use, distribution, and reproduction in any medium, provided the original author(s) and the source are credited.

\section{References}

[1] Le C, Bao M, Teo EY, et al. Advances in bone tissue engineering. In Regenerative Medicine and Tissue Engineering. Andrades JA, Ed. InTech, 2013, DOI: $10.5772 / 55916$.

[2] Nilsson M, Wang JS, Wielanek L, et al. Biodegradation and biocompatibility of a calcium sulphate-hydroxyapatite bone substitute. J Bone Joint Surg Br 2004, 86: 120-5.

[3] $\mathrm{Xu} \mathrm{HHK,} \mathrm{Simon} \mathrm{Jr.} \mathrm{CG.} \mathrm{Fast} \mathrm{setting} \mathrm{calcium}$ phosphate-chitosan scaffold: Mechanical properties and biocompatibility. Biomaterials 2005, 26: 1337-1348.

[4] Kikuchi $\mathrm{M}$, Itoh $\mathrm{S}$, Ichinose $\mathrm{S}$, et al. Self-organization mechanism in a bone-like hydroxyapatite/collagen nanocomposite synthesized in vitro and its biological reaction in vivo. Biomaterials 2001, 22: 1705-1711.

[5] Kikuchi M, Matsumoto $\mathrm{HN}$, Yamada $\mathrm{T}$, et al. Glutaraldehyde cross-linked hydroxyapatite/collagen self-organized nanocomposites. Biomaterials 2004, 25 : 63-69. 
[6] Kikuchi M, Ikoma T, Itoh S, et al. Biomimetic synthesis of bone-like nanocomposites using the self-organization mechanism of hydroxyapatite and collagen. Compos Sci Technol 2004, 64: 819-825.

[7] Zhang W, Liao SS, Cui FZ. Hierarchical self-assembly of nano-fibrils in mineralized collagen. Chem Mater 2003, 15: 3221-3226.

[8] Zhai Y, Cui FZ. Recombinant human-like collagen directed growth of hydroxyapatite nanocrystals. J Cryst Growth 2006, 291: 202-206.

[9] Olszta MJ, Cheng X, Jee SS, et al. Bone structure and formation: A new perspective. Materials Science and Engineering: R: Reports 2007, 58: 77-116.

[10] Yoon B-H, Kim H-W, Lee S-H, et al. Stability and cellular responses to fluorapatite-collagen composites. Biomaterials 2005, 26: 2957-2963.

[11] Kim H-W, Kim H-E, Salih V. Stimulation of osteoblast responses to biomimetic nanocomposites of gelatinhydroxyapatite for tissue engineering scaffolds. Biomaterials 2005, 26: 5221-5230.

[12] Costa HS, Mansur AAP, Pereira MM, et al. Engineered hybrid scaffolds of poly(vinyl alcohol)/bioactive glass for potential bone engineering applications: Synthesis, characterization, cytocompatibility, and degradation. Journal of Nanomaterials 2012, 2012: 718470.

[13] Wang YJ, Chen JD, Wei K, et al. Surfactant-assisted synthesis of hydroxyapatite particles. Mater Lett 2006, 60: 3227-3231.

[14] Wang Y, Zhang S, Wei K, et al. Hydrothermal synthesis of hydroxyapatite nanopowders using cationic surfactant as a template. Mater Lett 2006, 60: 1484-1487.

[15] Walsh D, Kingston JL, Heywood BR, et al. Influence of monosaccharides and related molecules on the morphology of hydroxyapatite. J Cryst Growth 1993, 133: 1-12.

[16] Yin YJ, Zhao F, Song XF, et al. Preparation and characterization of hydroxyapatite/chitosan-gelatin network composite. $J$ Appl Polym Sci 2000, 77: 2929-2938.
[17] Shou ZJ, Le HR, Qu SY, et al. Fabrication and mechanical properties of chitosan-montmorillonite nano-composite. Key Eng Mat 2012, 512-515: 1746-1750.

[18] Yan Y, Zhang X, Mao H, et al. Hydroxyapatite/gelatin functionalized graphene oxide composite coatings deposited on $\mathrm{TiO}_{2}$ nanotube by electrochemical deposition for biomedical applications. Appl Surf Sci 2015, 329: 76-82.

[19] Kim H-W, Knowles JC, Kim H-E. Hydroxyapatite and gelatin composite foams processed via novel freeze-drying and crosslinking for use as temporary hard tissue scaffolds. $J$ Biomed Mater Res A 2005, 72A: 136-145.

[20] Tadic D, Peters F, Epple M. Continuous synthesis of amorphous carbonated apatites. Biomaterials 2002, 23: 2553-2559.

[21] Huang Y, Zhang X, Mao H, et al. Osteoblastic cell responses and antibacterial efficacy of $\mathrm{Cu} / \mathrm{Zn}$ co-substituted hydroxyapatite coatings on pure titanium using electrodeposition method. RSC $A d v$ 2015, 5: 17076-17086.

[22] Huang Y, Yan Y, Pang X, et al. Bioactivity and corrosion properties of gelatin-containing and strontium-doped calcium phosphate composite coating. Appl Surf Sci 2013, 282: 583-589.

[23] Le HR, Chen KY, Wang CA. Effect of $\mathrm{pH}$ and temperature on the morphology and phases of co-precipitated hydroxyapatite. J Sol-Gel Sci Technol 2012, 61: 592-599.

[24] Koutsopoulos S. Synthesis and characterization of hydroxyapatite crystals: A review study on the analytical methods. J Biomed Mater Res 2002, 62: 600-612.

[25] Manickavasagam B. Extraction of gelatine. USPTO Patent Application 20080167447, 2008.

[26] Meyers MA, Chen P-Y, Lin AY-M, et al. Biological materials: Structure and mechanical properties. Prog Mater Sci 2008, 53: 1-206.

[27] Takeuchi A, Ohtsuki C, Miyazaki T, et al. Heterogeneous nucleation of hydroxyapatite on protein: Structural effect of silk sericin. J R Soc Interface 2005, 2: 373-378. 